\title{
Computational analysis of movement behaviors of medaka (Oryzias latipes) after the treatments of copper by using fractal dimension and artificial neural networks
}

\author{
C. W. Ji ${ }^{1}$, S. H. Lee ${ }^{2}$, I.-S. Kwak ${ }^{3}$, E. Y. Cha ${ }^{4}$, S.-K. Lee ${ }^{5}$ \\ \& T.-S. Chon ${ }^{1}$ \\ ${ }^{1}$ Division of Biological Sciences, \\ Pusan National University, Republic of Korea \\ ${ }^{2}$ Department of Physics, Pusan National University, Republic of Korea \\ ${ }^{3}$ Faculty of Marine Technology, \\ Chonnam National University, Republic of Korea \\ ${ }^{4}$ Department of Electronics Engineering, \\ Pusan National University, Republic of Korea \\ ${ }^{5}$ Toxicology Research Center, \\ Korea Research Institute of Chemical Technology, Republic of Korea
}

\begin{abstract}
Response behaviors of medaka were computationally analyzed before and after the treatments of copper at low concentration $(1.0 \mathrm{mg} / \mathrm{L})$. Parameters (e.g., speed, stop time, turning rate, etc) of the movement patterns were used as input for training the Multi-Layer Perceptron. Detection rates of the movement patterns such as 'Slow movement' and 'No movement' increased after the treatments. However, a higher degree of variation was observed in detection rates. Fractal dimension calculated from the movement data of individual specimens decreased consistently after the treatments. Higher consistency in fractal dimension was further achieved by using the data for collective rearing. Feasibility of behavioral monitoring was discussed in assessing toxic chemicals in environment.

Keywords: response behavior, medaka, copper, fractal dimension, Artificial Neural Network, behavioral monitoring.
\end{abstract}




\section{Introduction}

Recently automatic detection of response behaviors of animals has been considered as an efficient tool for bio-monitoring of aquatic ecosystems [1]. Dutta et al [2] suggested that a behavioral bioassay would be more sensitive than other types of testing methods. A numerous accounts of behavioral research on effects of toxic chemicals at low concentrations have been reported in various taxa, including crustaceans [3, 4], snails [5], fish [6] and insects [7, 8]. Recently Oshima et al [9] observed suppression of sexual behavior in male medaka exposed to estradiol. However, these studies are mostly based on observation of single or combinations of single behaviors mainly with qualitative descriptions. Not much computational research has been carried out for automatically detecting behavioral changes from continuous recording.

Behaviors, however, have been regarded as difficult for analysis due to complexity residing in the data. Theoretical studies have been carried out on analyzing movement data regarding correlation function [10], random walk [11], etc. Recently fractal dimension has been considered as an efficient parameter to quantitatively express behavioral states. Fractal dimension has been widely used for analyzing non-linear phenomena in biological and ecological sciences such as geographical features, morphology, etc, [see 12]. Johnson et al [13] and Weins et al [14, 15] used fractal dimension for analysis of insect movement to quantitatively characterize behavioral states that might not be available through absolute measures of pathway configurations. Alados et al [16] used fractal dimension for detecting response behaviors of parasitic infection in Spanish ibex. In this study we used fractal dimension to reveal behavioral states of indicator specimens in response to toxic substances.

Along with fractal dimension, we also implemented Artificial Neural Networks (ANNs) to address pattern changes in response behaviors. While fractal dimension quantitatively compresses behavioral changes as one parameter, ANNs are useful for dealing with local information and for revealing specific behavioral patterns explicitly. ANNs have been widely used for analyzing complex data in computer and electronics engineering [see 17, 18] and have been recently implemented to ecological sciences in various aspects such as forecasting, input-output relationships, data organization, classification, etc [see 19, 20]. Recently ANNs have been applied to behavioral monitoring. SelfOrganizing Map was applied to classification of response behaviors of indicator organisms treated with diazinon [21, 22]. Multi-Layer Perceptron (MLP) was used to automatically detect behavioral changes in organisms such as medakas and chionomids in response to toxic chemicals [23, 24].

In this study we intend to extract local and global information residing in behavioral data and to propose a system to quantitatively characterize response behaviors in both explicit (i.e., MLP) and compressed (i.e., fractal dimension) forms. Initially MLP was applied to detection of changes in specific movement patterns after the treatments of toxic substances. Subsequently we elucidated fractal dimension as a means of minimizing the variability of behavioral data to be a reliable parameter to detect changes in behavioral states. 


\section{Materials and methods}

\subsection{Test specimens and observation system}

Medakas (Oryzias latipes), the "or" strain originally developed by Bioscience Center, Nagoya University, Japan, were obtained from Toxicology Research Center, Korea Research Institute of Chemical Technology (KRICT; Taejeon, Korea) for testing. The stock populations were maintained in a glass tank, and were reared with artificial dry diet $\left(\right.$ Tetramin $\left.^{\circledR}\right)$ under the light regime of L10:D14 in temperature ranging $25 \pm 1{ }^{\circ} \mathrm{C}$. In photo-phase, a fluorescent lamp $(20 \mathrm{~W})$ was used as the light source and was located above the observation aquarium with $30 \mathrm{~cm}$ apart. In scoto-phase, a red light $(20 \mathrm{~W})$ was provided at the same position.

The position of the test specimens of medaka (age: 6-12 months) was recorded by using an observation system consisting of an observation aquarium, a camera and software for image recognition. Individuals or groups of medakas were placed in a glass aquarium (volume of water: $40 \mathrm{~cm} \times 20 \mathrm{~cm} \times 10 \mathrm{~cm}$ ), and their position was scanned from the side view at $0.25 \mathrm{~s}$ intervals using a CCTV camera (Kukjae Electronics Co. Ltd.; IVC-841 ${ }^{\circledR}$ ) for four days (two days before the treatments and two days after the treatments). The analog data captured by the camera were digitized by using a video overlay board (Sigmacom Co., LTD.; Sigma TV $\mathrm{II}^{\mathbb{B}}$ ), and were sent to the image processing system to locate the target organisms in two dimension. The software for recognition of the movement tracks and other supporting mathematical programs were provided according to [23].

During the period of observation, disturbances in experimental conditions were minimized: oxygen, fresh water and food were not supplied to test specimens to simplify observation conditions [23]. Before monitoring, the specimens were acclimated to the observation aquarium for 1-2 days. Environmental factors such as light and temperature were maintained to the same condition for rearing stock populations.

\subsection{Experimental procedure}

Copper was treated to medaka fishes in this study. The level of $\mathrm{LC}_{50}$ for copper to medaka population was reported as $5 \mathrm{mg} / \mathrm{L}$ [25]. After two days of observation without treatment, reaction behaviors were also recorded for two days after the treatments of copper $(1 \mathrm{mg} / \mathrm{L})$.

Initially, we observed 10 medaka fishes individually before and after the treatments. The parameters were extracted from the segmented data in every $30 \mathrm{~s}$. Based on previous research on the movement tracks [23], the following parameters were selected to characterize the movement patterns and were subsequently used as input data for training MLP (see section 2.3):

1) Speed $(\mathrm{mm} / \mathrm{s})$ : average in movement distance of the fish during the observation time. 
2) $Y$-position $\left(\mathrm{mm} / \mathrm{sec}^{2}\right)$ : the average distance in $Y$-axis measured from the surface during the observation time; as the specimens was located close to the surface, the $Y$-position was decreased

3) Stop number: the total frequency in which specimen did not move.

4) Stop duration (total time of stops: s): the total duration in which the specimen did not move.

5) Turning rate ( $\mathrm{rad} / \mathrm{s})$ : the sum of angle changes in radian in absolute values divided by the cumulated time duration of movement.

6) Meander ( $\mathrm{rad} / \mathrm{mm})$ : the sum of angle changes in radian in absolute values divided by the path length.

For calculating statistics of the parameters of the movement tracks, we selected 10 sample segments $(30 \mathrm{~s})$ by visual observation for each movement pattern. This process was repeated for 10 specimens. In total 100 segments were obtained for each pattern. Among the selected samples 30 segments were randomly chosen for statistical analysis. Subsequently 10 samples were independently selected for the MLP training by random sampling. For testing the trained MLP, the whole sequence of the movement data for four days (2 days separately for each 'before' and 'after' the treatments) were provided to the trained MLP. The data segments in every $30 \mathrm{~s}$ interval were continuously provided to be recognized by the trained network.

Medaka fishes were also reared in groups with four specimens in the same conditions applied to individual rearing in 10 replications. Fractal dimension (see section 2.4) was calculated for the movement data for specimens in individual and group rearing.

\subsection{Multi-Layer Perceptron (MLP)}

The MLP [26] was trained with the data for the movement tracks. Training proceeds to minimize the mean square error between the actual input and desired output (or target value) according to the back-propagation algorithm (Fig. 1) [18, 26]. In this study the parameters characterizing the movement tracks were used as input data (6 nodes), while the decision of the movement patterns were given in the binary form as matching output (6 nodes).

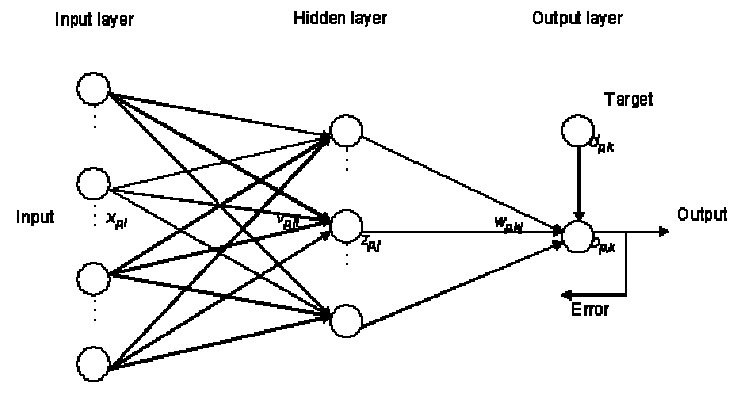

Figure 1: $\quad$ The schematic diagram of MLP. 
The net input $\left(N E T_{p, j}\right)$ to neuron $j$ of the hidden layer for pattern $p$ is calculated as the summation of each input layer output $\left(X_{p} i\right.$; input value of parameter) multiplied by weight $\left(v_{j i}\right)$. The similar calculation is provided for the neuron $k$ of the output layer being linked by summation of each hidden layer output $\left(Z_{p, j}\right)$. An activation function (logistic function in this case) is applied to calculate the output of neuron $j$ of the hidden layer $\left(Z_{p, j}\right)$ and the output of neuron $k$ of the output layer $\left(O_{p, k}\right)$, according to the following eqn. (1):

$$
f(N E T)=\frac{1}{1+\exp (-\lambda N E T)}
$$

where $\lambda$ is the activation function coefficient. NET is expressed either in $Z_{p, j}$ or $O_{p, k}$ as follows, eqn. (2), (3):

$$
\begin{gathered}
z_{p, j}=f\left(\sum_{i} x_{p, i} v_{j i}\right) \\
o_{p, k}=f\left(\sum_{j} z_{p, j} w_{k j}\right)
\end{gathered}
$$

where $v_{j i}$ and $w_{k, j}$ are the connection weight between neuron $i$ of the input layer and neuron $j$ of the hidden layer, and the connection weight between neuron $j$ of the hidden layer and neuron $k$ of the output layer, respectively.

The back-propagation algorithm adjusts the connection intensities (weights $\left(v_{j i}\right)$ and $\left.\left(w_{k, j}\right)\right)$ of the network in a way that minimizes error. The sum of the errors in each neuron for pattern $p, E r r_{p}$, is calculated as follows, eqn. (4):

$$
\operatorname{Err}_{p}=\frac{1}{2} \sum_{k}\left(d_{p, k}-o_{p, k}\right)^{2}
$$

where $d_{p, k}$ is the target value corresponding to pattern $p$ at neuron $k$. The value of the activation function coefficients, $\lambda$, used in this study was 1.0 , and the learning coefficient, which updates the weights in iterative calculation, was set at 0.01 . The level of error tolerance was 1.0, and the threshold for determining the binary level for the activation function was 0.5. Network pruning was not required during the training process in this study. Details of using MLP can be found in the related bibliographies $[17,18,26,27]$.

\subsection{Fractal dimension}

Fractal dimension, $D$, was measured on location of specimens in individual rearing. The points recorded in every $0.25 \mathrm{sec}$ in 1-hour segment of the movement tracks were used for calculation based on the Box-Counting method (MATLAB $^{\circledR}$ 5.3.), eqn. (5):

$$
N(r)=(1 / r)^{D}, \quad D=\frac{\log N}{\log (1 / r)}
$$

where $N(r)$ is the number of points observed within the box sized as $r^{2}$. The two values, $N(r)$ and $r$ are presented as a linear form by the double logarithmic graph. 
In each 1-hour segment of the movement tracks, overlapping was allowed for $30 \mathrm{~min}$.

Fractal dimension was also measured for the data from group rearing. The data points $(0.25 \mathrm{~s}$ interval) of four specimens in the 15 -minute segments were used for calculating fractal dimension. In each segment of the movement tracks overlapping was allowed for $30 \mathrm{~min}$ and $7.5 \mathrm{~min}$ for individual and group rearing respectively.

\section{Results}

\subsection{Characterization of behavioral patterns}

Behaviors of fish have been reported to show typical patterns, including stationary movement, up-down swimming with circular motion, eating, agonistic behavior, hiding, etc [28]. In this research we also observed some clear movement patterns of medakas under the experimental conditions. Figure 2 shows the typical movement patterns of the tested specimens.

(a)

(c)

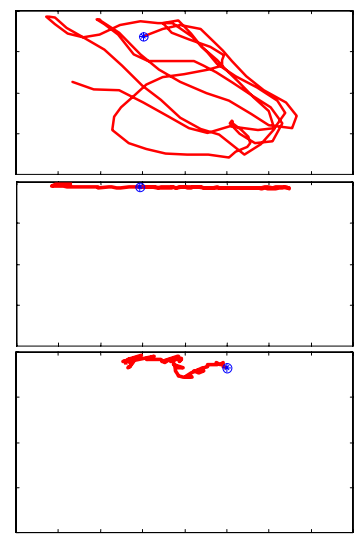

(b)

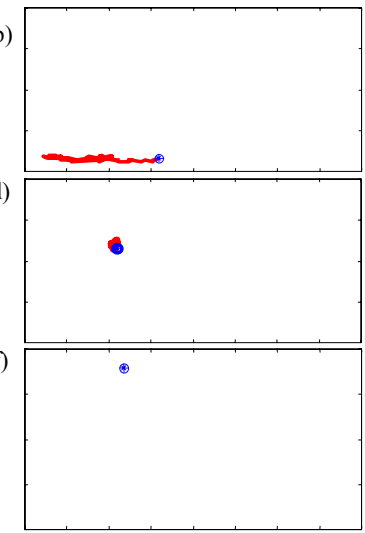

Figure 2: $\quad$ The movement tracks (side view) showing the behavioral patterns of medaka specimens in $30 \mathrm{~s}$ segments (a); Swimming, (b); Feeding, (c); Surface movement, (d); Slow movement, (e); Frequent stop, (f); No movement).

'Swimming' presented the active state of specimens (Fig. 2(a)), being characterized by the highest speed $(65.82 \mathrm{~mm} / \mathrm{s})$ and wide circling in the observation aquarium (Table 1). 'Feeding' showed the horizontal movement along the bottom of the observation aquarium in the limited range (Fig. 2(b)). The $Y$-position $(180.02 \mathrm{~mm})$ of 'Feeding' is higher than any other patterns (Table 1). In the observation system, the position on $Y$-coordinate movement is higher as it is closer to the bottom of the aquarium. 'Surface movement' showed horizontal activity near the top area of the aquarium (Fig. 2(c)). In contrast to the 
'Feeding', $Y$-position (15.56 mm) of 'Surface movement' was lower than any other patterns (Table 1). 'Slow movement' showed the lower phase of activity: the stop time (20.92 s) was longer than any other patterns (Table 1). 'Stop' is defined as the specimens maintaining the same position for the duration of $0.25 \mathrm{~s}$ in this study. Stop time is calculated as summation of the time duration for each stop. 'Frequent stop' is another pattern showing slow phase of activity. The specimens repeated the 'stop' and 'short advancement'. Stop number in 'Frequent stop' was observed as frequently as in 'Slow movement', however stop time was distinctively shorter in 'Frequent stop' (6.2 s) than in 'Slow movement' (20.92 s) (Table 1). Overall, 'Frequent stop' presented somewhat more active states compared with 'Slow movement'.

Table 1: Parameters characterizing the different movement patterns of medaka specimens before and after the treatments of copper $(n=30$ for each parameter for each pattern, (a); Swimming, (b); Feeding, (c); Surface movement, (d); Slow movement, (e); Frequent stop).

\begin{tabular}{cccccccccccccc}
\hline & \multicolumn{1}{c}{$\begin{array}{c}\text { Speed } \\
(\mathrm{mm} / \mathrm{s})\end{array}$} & $\begin{array}{c}\text { Y-position } \\
(\mathrm{mm})\end{array}$ & $\begin{array}{c}\text { Stop time } \\
(\mathrm{sec})\end{array}$ & $\begin{array}{c}\text { Stop } \\
\text { number }\end{array}$ & $\begin{array}{c}\text { Turning rate } \\
(\mathrm{rad} / \mathrm{s})\end{array}$ & $\begin{array}{c}\text { Meander } \\
(\mathrm{rad} / \mathrm{mm})\end{array}$ \\
\cline { 2 - 13 } Patterns & Mean & SD & Mean & SD & Mean & SD & Mean & SD & Mean & SD & Mean & SD \\
\hline a & 65.8 & 12.4 & 90.7 & 16.4 & 0.3 & 0.5 & 0.7 & 1.2 & 1.7 & 0.3 & 0.0 & 0.0 \\
b & 11.2 & 2.4 & 180.2 & 3.4 & 7.7 & 4.0 & 11.7 & 2.7 & 4.4 & 0.8 & 0.3 & 0.1 \\
$\mathrm{c}$ & 17.5 & 9.8 & 15.6 & 7.2 & 7.2 & 5.0 & 11.2 & 6.0 & 2.9 & 1.2 & 0.2 & 0.1 \\
$\mathrm{~d}$ & 3.6 & 1.1 & 97.9 & 82.1 & 20.9 & 3.3 & 13.1 & 4.8 & 5.9 & 1.0 & 0.5 & 0.1 \\
$\mathrm{e}$ & 10.0 & 2.0 & 107.6 & 39.3 & 6.2 & 3.5 & 14.2 & 4.8 & 5.0 & 1.4 & 0.4 & 0.1 \\
\hline
\end{tabular}

Table 2: Analysis of variance (ANOVA) and Tukey test for multiple comparisons of parameters characterizing the different movement patterns of medaka specimens before and after the treatments $(n=30$ for each parameter for each pattern, (a); Swimming, (b); Feeding, (c); Surface movement, (d); Slow movement, (e); Frequent stop).

\begin{tabular}{cccc}
\hline Parameters & $\mathrm{F}^{1}$ & $\mathrm{P}$ & $\begin{array}{c}\text { Comparison of parameters } \\
\text { (Tukey test, } \alpha=0.05)\end{array}$ \\
\hline Speed $(\mathrm{mm} / \mathrm{s})$ & 368.01 & $<0.001$ & $\mathrm{a} \neq \mathrm{c} \neq \mathrm{b}=\mathrm{e} \neq \mathrm{d}$ \\
Y-position $(\mathrm{mm})$ & 59.38 & $<0.001$ & $\mathrm{~b} \neq \mathrm{e}=\mathrm{d}=\mathrm{a} \neq \mathrm{c}$ \\
Stop time $(\mathrm{sec})$ & 133.75 & $<0.001$ & $\mathrm{~d} \neq \mathrm{b}=\mathrm{c}=\mathrm{e} \neq \mathrm{a}$ \\
Stop number & 48.99 & $<0.001$ & $\mathrm{e}=\mathrm{d}=\mathrm{b}=\mathrm{c} \neq \mathrm{a}$ \\
Turning rate $(\mathrm{rad} / \mathrm{s})$ & 80.11 & $<0.001$ & $\mathrm{~d} \neq \mathrm{e}=\mathrm{b} \neq \mathrm{a}=\mathrm{c}$ \\
Meander $(\mathrm{rad} / \mathrm{mm})$ & 112.23 & $<0.001$ & $\mathrm{~d} \neq \mathrm{e}=\mathrm{b} \neq \mathrm{c}=\mathrm{a}$ \\
\hline${ }^{1} \mathrm{~F}$ 0.05(2), $5,150=2.66$ & &
\end{tabular}


The parameters mostly appeared to be statistically different in different movement patterns according to the Tukey test [29] (Table 2). Regarding speed, 'Swimming', 'Surface movement' and 'Slow movement' were different among the patterns, but 'Frequent stop' and 'Feeding' were in the same range. 'Feeding' and 'Frequent stop' were also similar in the other parameters including stop time, stop number, turning rate and meander. $Y$-position, however, was different between the two patterns. The other movement patterns were uniquely distinguished each other and were statistically different (Table 2).

\subsection{MLP applied to individual data}

The parameters characterizing the movement patterns were effectively learned by MLP with the training rates mostly over $92 \%$. As stated before the whole data set was used for testing. The pattern of input segment $(30 \mathrm{~s})$ was recognized by the trained MLP. Detection rates were calculated as the number of correct recognition divided the number of the total recognition for each individual. The detection rate for each specimen was subsequently averaged with 10 specimens. Table 3 shows changes in detection rates $(\%)$ for each pattern in averages for 10 specimens before and after the treatments.

Table 3: Detection rate (\%) of different movement patterns of medaka specimens before and after the treatment of copper (a; Swimming, b; Feeding, c; Surface movement, d; Slow movement, e; Frequent stop, f; No movement). $(n=10)$.

\begin{tabular}{cccccccc}
\hline \multirow{2}{*}{ Treatments } & \multirow{2}{*}{ Patterns } & \multicolumn{2}{c}{ Day } & \multicolumn{2}{c}{ Night } & \multicolumn{2}{c}{ All } \\
\cline { 3 - 8 } & & Mean & SD & Mean & SD & Mean & SD \\
\hline \multirow{3}{*}{ Before } & a & $31.48 \%$ & 30.21 & $10.79 \%$ & 17.93 & $21.13 \%$ & 26.41 \\
Treatment & b & $13.10 \%$ & 11.24 & $7.91 \%$ & 6.84 & $10.50 \%$ & 9.44 \\
& c & $6.56 \%$ & 10.21 & $7.74 \%$ & 5.07 & $7.34 \%$ & 7.73 \\
& d & $5.80 \%$ & 5.25 & $10.70 \%$ & 5.68 & $8.25 \%$ & 5.89 \\
& e & $4.24 \%$ & 3.75 & $9.12 \%$ & 9.12 & $6.68 \%$ & 7.23 \\
& f & $6.19 \%$ & 7.89 & $20.26 \%$ & 19.55 & $13.22 \%$ & 16.21 \\
\cline { 2 - 8 } After Treatmen & a & $12.51 \%$ & 13.25 & $3.51 \%$ & 3.49 & $8.01 \%$ & 10.5 \\
& b & $8.21 \%$ & 5.93 & $3.23 \%$ & 2.72 & $5.72 \%$ & 5.17 \\
& c & $3.56 \%$ & 3.94 & $2.45 \%$ & 1.72 & $3.01 \%$ & 3.01 \\
& d & $14.91 \%$ & 7.33 & $19.45 \%$ & 5.18 & $17.18 \%$ & 6.6 \\
& d & $9.21 \%$ & 5.87 & $8.77 \%$ & 5.81 & $8.99 \%$ & 5.69 \\
& f & $21.88 \%$ & 11.37 & $37.93 \%$ & 9.41 & $29.21 \%$ & 13.07 \\
\hline
\end{tabular}

Before the treatments, detection rate of 'Swimming' pattern was high with $21.13 \%$, but the rate decreased to $8.01 \%$ after the treatments (Table 3). The 'Feeding' (from 10. 50\% to 5.72\%) and 'Surface movement' (from 7.34\% to 
$3.01 \%$ ) patterns also accordingly decreased. In contrast, detection rates for 'Slow movement' (from $8.25 \%$ to $17.18 \%$ ), 'Frequent stop' (form $6.68 \%$ to $8.99 \%$ ) and 'No movement' (from 13.22\% to 29.21\%) increased after the treatments of copper (Table 3). In general, detection rates for the patterns representing high activity (e.g., 'Swimming', 'Feeding' etc) were decreased after the treatments.

Higher variation was observed in the detection rates obtained by the trained network. Standard deviations in detection rates were in the higher range, and all the parameters were not distinguished with statistical tests. Table 4 shows the comparison of detection rates $(\%)$ before and after the treatments based on the $t$ test ( $n=10$ for each pattern) in different light phases. The patterns of 'Slow movement' and 'No movement' were statistically significant accordingly in photo- and scoto-phase, and the total periods (Table 4). Although the average values showed differences, 'Swimming', 'Feeding' and 'Frequent stop' were not statistically different before and after the treatments. The trends of behavioral changes were similar in scoto- and photo-phases. 'Surface movement' was only different at night before and after the treatments (Table 4).

Table 4: Comparison of detection rates $(\%)$ in different movement patters before and after the treatments based on the t-test $(n=10$ for each pattern, a; Swimming, b; Feeding, c; Surface movement, d; Slow movement, e; Frequent stop, f; No movement).

\begin{tabular}{ccccccc}
\hline & \multicolumn{2}{c}{ Photophase } & \multicolumn{2}{c}{ Scotophase } & \multicolumn{2}{c}{ Total period } \\
\cline { 2 - 7 } & $\mathrm{t}$ & $P$ & $\mathrm{t}$ & $P$ & $\mathrm{~T}$ & $P$ \\
\hline $\mathrm{a}$ & 1.818 & n.s. & 1.259 & n.s. & 1.666 & n.s. \\
$\mathrm{b}$ & 1.215 & n.s. & 2.012 & n.s. & 1.646 & n.s. \\
$\mathrm{c}$ & 0.992 & n.s. & 3.123 & $0.01<P<0.02$ & 1.815 & n.s. \\
$\mathrm{d}$ & 3.193 & $0.01<P<0.02$ & 3.599 & $0.005<P<0.01$ & 3.836 & $0.002<P<0.005$ \\
$\mathrm{e}$ & 2.255 & n.s. & 0.100 & n.s. & 0.912 & n.s. \\
$\mathrm{f}$ & 3.587 & $0.005<P<0.01$ & 2.576 & $0.05<P<0.02$ & 3.293 & $0.005<P<0.01$ \\
\hline
\end{tabular}

$\mathrm{t}_{0.05(2), 9}=2.262$

\subsection{Fractal dimension applied to individual rearing}

In contrast to the results from MLP, fractal dimension showed more consistency in revealing changes in behavioral states of medaka specimens after the treatments of copper (Fig. 3). Although there were individual variations, decrease in fractal dimension appeared consistently for all the tested specimens. The average in fractal dimension was $1.62 \pm 0.10$ before the treatments, but decreased to $1.42 \pm 0.16$ after the treatments. The Nested ANOVA indicated that the values of fractal dimension were statistically different between 'before' and 'after' the treatments ( $\mathrm{df}=(1,18), \mathrm{F}=6.2,0.02<P<0.05)$. The sub-group of individual specimens, however, was different $(\mathrm{df}=(18,600), \mathrm{F}=3.07$, $P<0.001)$. This indicated that individual variation existed in the values of fractal dimension. 


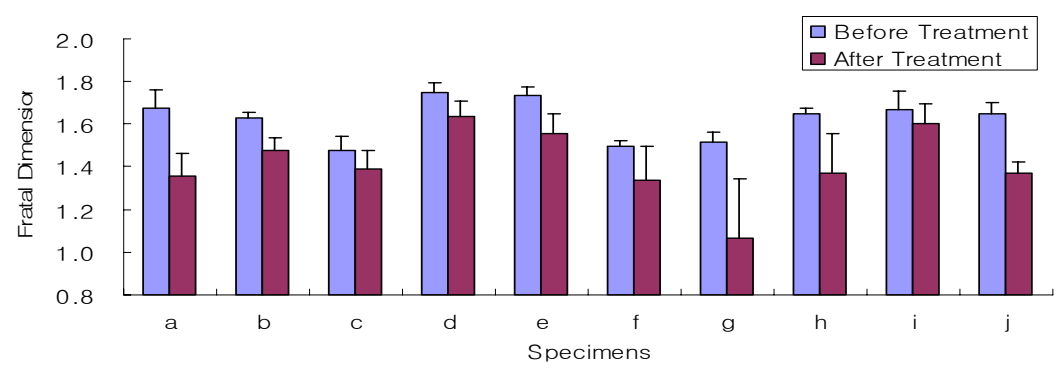

Figure 3: Fractal dimension of the movement points in different specimens of medaka obtained from individual rearing before and after the treatments of copper, $1 \mathrm{mg} / \mathrm{L}$.

\subsection{Fractal dimension applied to group rearing}

We further analyzed fractal dimension of the movement points when the specimens were reared in groups of 4 specimens (Fig. 4). Fractal dimension consistently decreased after the treatments of copper $(1 \mathrm{mg} / \mathrm{L})$ in different groups, being similar to the case of individual rearing. The average of fractal dimension was $1.63 \pm 0.02$ before the treatments and $1.46 \pm 0.07$ after the treatments. The values of fractal dimension from group rearing were more consistent compared with individual rearing. The Nested ANOVA showed that the values of fractal dimension were significantly different between the treatments $(\mathrm{df}=(1,18), \mathrm{F}=23.35, P<0.001)$. In contrast to the case of individual rearing, however, the values of fractal dimension were also in the similar range between the tested groups: the sub-group difference was not significant $(\mathrm{df}=(18$, $820), F=0.05, P>0.5)$. This indicated that individual variation in fractal dimension could be minimized through group rearing of fishes.

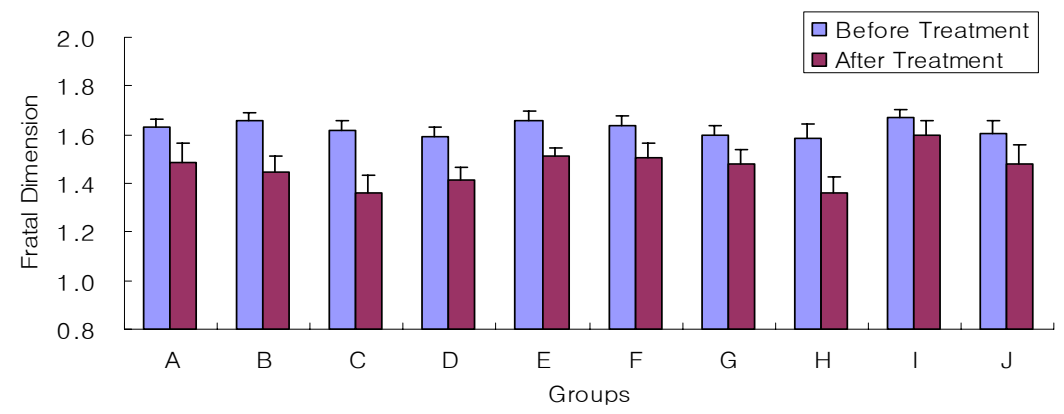

Figure 4: Fractal dimension of the movement points in different groups of the 4 medaka specimens before and after the treatments of copper, $1.0 \mathrm{mg} / \mathrm{L}$. 


\section{Discussion and conclusions}

A computational system was developed for automatically detecting the movement states of medaka specimens in this study. Although individual variation occurred, MLP was useful for detecting movement patterns explicitly. The specific patterns such as 'Slow movement' and 'No movement' were statistically different before and after the treatments (Table 3). These patterns could be used as indicator patterns of medakas for detecting presence of toxic substance in environment.

We further showed that the higher variation in individuals could be decreased by using fractal dimension. The values of fractal dimension appeared to consistently decrease for all the tested specimens after the treatments (Fig. 3). The group testing, consisting of 4 medaka fishes, further minimized the variation of fractal dimension by showing no statistical difference among different groups (Fig. 4). Consistency in the measurement of fractal dimension was revealed in comparing Coefficient of Variation (CV: standard deviation divided by mean) (Fig. 5). CVs for group rearing were lower for both 'before' and 'after' the treatments. The difference between individual and group rearing was more clearly observed after the treatments with the statistical significance $(\mathrm{df}=(1,18)$, $\mathrm{F}=6.01,0.02<P<0.05)$. The statistical difference was not observed for CVs between individual and group rearing before the treatments $(\mathrm{df}=(1,18)$, $\mathrm{F}=0.66, P>0.5$ ), however the average value was lower for group rearing (Fig. 5).

This study indicated that fractal dimension based on group rearing could be used as a reliable parameter to indicate behavioral changes of medakas after the treatments of copper. Another advantage of fractal dimension is the flexibility in recording data points in group rearing. In the image processing system, it is in general difficult to trace the movement tracks for each specimen in group rearing especially if the specimens are small in size. Fractal dimension, however, was measured from the positions of the specimens collectively, and tracing each individual movement was not necessary in this case.

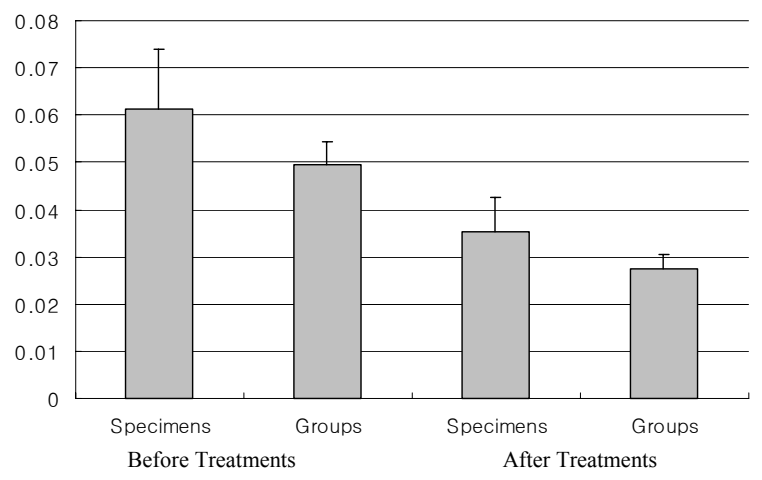

Figure 5: Comparison of CVs of fractal dimension in individual and group rearing before and after the treatments of copper, $1.0 \mathrm{mg} / \mathrm{L}$. 
In real situation, MLP and fractal dimension could be used in combination for providing more practical information for warning system in risk assessment. While fractal dimension would provide information on the global change in behavioral states more consistently in a compressed form, MLP would reveal differences in the specific patterns, thus providing more detailed information on explicit response behaviors. The two methods could be combined to produce an efficient monitoring system for in-situ risk assessment in aquatic systems in the future.

In this study we used copper as toxic substances. Copper plays an essential role in mitochondrial function, detoxification of free radicals, neurotransmitter synthesis, cross-linking of connective tissue, and cellular iron metabolism. Copper causes mutations in genes encoding "P-type" transport ATPase and induces neurotic disease such as Lou Gehrig's and Wilson's disease [30]. The toxicological impact would consequently produce stressful responding behaviors of the organisms. Toxic responses to copper have been reported on some indicator species. Activity accordingly decreased in Daphnia magna and Gammarus [31, 32]. Not much quantitative research, however, has been conducted on behavioral changes especially on vertebrates such as fish. In this study, we demonstrated that computational methods such as MLP and fractal dimension could be efficiently used for monitoring contamination of copper by using fish as indicator specimens.

In conclusion, MLP could accommodate local information on response behaviors and would be useful for detecting changes in specific patterns. The consistency in behavioral detection was achieved by fractal dimension especially through group rearing, and the parameter could be useful source of a reliable indicator in determining behavioral states of specimens exposed to toxic chemicals. MLP and fractal dimension could be used in combination as an efficient means of in-situ monitoring by providing both 'local and more specific' (i.e., MLP), and 'global and more consistent' (i.e., fractal dimension) information concurrently.

\section{Acknowledgements}

This work was supported by grant No. R01-2004-000-11036-0 from the Basic Research Program of the Korea Science \& Engineering Foundation. We appreciate provision of the original stock for test specimens from the Bioscience Center, Nagoya University, Japan.

\section{References}

[1] Lemly, A.D. \& Smith, R.J., A behavioral assay for assessing effects of pollutants of fish chemoreception. Ecotoxicology and Environmental Safety 11, pp. 210-218, 1986.

[2] Dutta, H., Marcelino, J. \& Richmonds, Ch., Brain acetylcholinesterase activity and optomotor behavior in bluegills, Lepomis macrochirus 
exposed to different concentrations of diazinon. Arch. Intern. Physiol. Biochim. Biophys. 100, pp. 331-334, 1992.

[3] Abgrall, P., Rangeley, R.W., Burridge, L.E. \& Lawton, P., Sublethal effects of azamethiphos on shelter use by juvenile lobsters (Homarus americanus). Aquaculture 181, pp. 1-10, 2000.

[4] Roast, S.D., Widdows, J. \& Jones, M.B., Disruption of swimming in the hyperbenthic mysid Neomysis integer (Peracarida: Mysidacea) by the organophosphate pesticide chlorpyrifos. Aquatic Toxicology 47, pp. 227$241,2000$.

[5] Ibrahim, W.L.F., Furu, P., Ibrahim, A.M. \& Christensen, N.Ø., Effect of the organophosphorous insecticide, chlorpyrifos (Dursban), on growth, fecundity and mortality of Biomphalaria alexandrina and on the production of Schistosoma mansoni cercariae in the snail. Journal of Helminthology 66, pp. 79-88, 1992

[6] Gray, M.A., Teather, K.L. \& Metcalfe, C.D., Reproductive success and behavior of Japanese Medaka (Oryzias latipes) exposed to 4-tertoctylphenol. Environmental Toxicology and Chemistry 18, pp. 25872594, 1999.

[7] Chon, T.-S., Park, Y.S. \& Ross, M.H., Activity of German cockroach, Blattella germanica (L.) (Orthoptera: Blattellidae), at different microhabitats in semi-natural conditions when treated with sublethal doses of pesticides. Journal of Asia-Pacific Entomology 1, pp. 99-107, 1998a.

[8] Chon, T.-S., Park, Y.S. \& Ross, M.H., Temporal pattern of local activity at harborage in two strains of the German cockroach, Blattella germanica (L.), in semi-natural conditions after treated with sublethal doses of insecticides. Korean Journal of Entomology 28, pp. 77-83, $1998 \mathrm{~b}$.

[9] Oshima, Y., Kang, I. J., Kobayashi, M., Nakayama, K., Imada, N. \& Honjo, T., Suppression of sexual behavior in male Japanese medaka (Oryzias latipes) exposed to 17ß-estradiol. Chemosphere 50, pp. 429-436, 2003.

[10] Alt, W., Correlation analysis of two-dimensional locomotion paths. In: Alt, W., Hoffmann, G. (Eds.), Biological Motion. Lecture Notes in Biomathematics 89, pp. 254-268, 1989.

[11] Berg, H. C., Random Walks in Biology. Trends in Neurosciences. 1983.

[12] Rybaczuk, M. \& Zieliński, W., The concept of physical and fractal dimension I. The projective dimensions. Chaos, Solitons, \& Fractals 12, pp. 2517-2535, 2001.

[13] Johnson, A.R., Milne, B.T. \& Wiens, J.A., Diffusion in fractal landscapes: simulations and experimental studies of tenebrionid beetle movements. Ecology 73, pp. 1968-1983, 1992.

[14] Wiens, J.A., Crist, T.O. \& Milne, B.T., On quantifying insect movement. Environmental Entomology 22, pp. 709-715, 1993.

[15] Weins, J.A., Crist, T.O., With, K.A. \& Milne, B.T., Fractal patterns of insect movement in microlandscape mosaics. Ecology 79, pp. 663-666, 1995. 
[16] Alados C. L., Escos J. M. \& Emlen J. M., Fractal structure of sequential behaviour patterns: an indicator of stress. Animal Behaviour, 51, pp. 437443, 1996.

[17] Lippmann, R.P., An Introduction to Computing with Neural Nets. IEEE ASSP Magazine, April. pp. 4-22, 1987.

[18] Zurada, J.M., Introduction to Artificial Neural Systems. West Publishing Company, New York. 1992.

[19] Chon, T.-S., Park, Y.S., Moon, K.H. \& Cha, E.Y., Patternizing communities by using an artificial neural network. Ecological Modeling 90, pp. 69-78, 1996.

[20] Lek, S., Delacoste, M., Baran, P., Dimopoulos, I., Lauga, J. \& Aulagnier, S., Application of Neural Networks to modelling nonlinear relationships in Ecology. Ecological Modelling 90, pp. 39-52, 1996.

[21] Chon, T.-S., Park, Y.-S., Park, J. Y., Choi, S.-Y., Kim, K. T. \& Cho, E. C., Implementation of computational methods to pattern recognition of movement behavior of Blattella germanica (Blattaria: Blattellidae) treated with Ca2+ signal inducing chemicals. Appl. Entomol. Zool. 39, pp. 79-96, 2004.

[22] Park, Y.-S., Chung, N.-I., Choi, K.-H. Cha, E. Y., Lee, S.-K. \& Chon, T.S., Computational characterization of behavioral response of medaka (Oryzias latipes) treated with diazinon. Aquatic Toxicology 71, pp. 215228, 2005.

[23] Kwak, I.-S., Chon, T. S., Kang, H. M., Chung, N. I., Kim, J. S., Koh, S. C., Lee, S. K. \& Kim, Y. S., Pattern recognition of the movement tracks of medaka (Oryzias latipes) in response to sub-lethal treatments of an insecticide by using artificial neural networks. Environmental Pollution 120, pp. 671-681, 2002.

[24] Kim, C.-K. \& Kwak, I.-S., Implementation of wavelets and artificial neural networks to detection of toxic response behavior of chironomids (Chironomidae: Diptera) for water quality monitoring. Ecological Modelling 195, pp. 61-71, 2006.

[25] Environmental Protection Agency., Ambient aquatic life water quality for copper. http://www.epa.gov/ost/pc/ambientwqc/copper1984.pdf., 1984.

[26] Rumelhart, D.E., Hinton, G.E. \& Williams, R.J., Learning internal representations by error propagation, in Parallel distributed processing: explorations in the microstructure of cognition. In: Rumelhart, D.E., McClelland, J.L. (Eds.), MIT Press, Cambridge MA. pp. 318 - 362, 1986.

[27] Haykin, S., Neural Networks. Macmillan College Publishing Company, New York. 1994.

[28] Hosn, W. A., Quantitative analysis and modelling of the behavioural dynamics of Salvelinus fontinalis (brook trout), Behavioural Processes. 6, pp. 105-120, 1999.

[29] Zar, J.H., Biostatistical Analysis. Prentice-Hall International, Englewood Cliffs. 1984.

[30] Wessling-Resnick, M., Understanding copper uptake at the molecular level. Nutrition Reviews. 60, pp. 177-179, 2002. 
[31] Untersteiner, H., Kahapka, J. \& Kaiser, H., Behavioural response of the cladoceran Daphnia magna to sublethal Copper stress - validation by image analysis. Applied Toxicology 65, pp. 435-442, 2003.

[32] Mills, C. L., Shukla, D. H. \& Compton, G. J., Development of a new low cost high sensitivity system for behavioural ecotoxicity testing. Aquatic Toxicology 77, pp. 197-201, 2006. 